\title{
Pre-perihelion activity of comet 67P/Churyumov-Gerasimenko ${ }^{\star}$
}

\author{
A. Guilbert-Lepoutre ${ }^{1}$, R. Schulz ${ }^{1}$, A. Rożek ${ }^{2}$, S. C. Lowry ${ }^{2}$, G. P. Tozzi ${ }^{3}$, and J. A. Stüwe ${ }^{4,5}$ \\ 1 European Space Agency - ESTEC, 2200 AG Noordwijk, The Netherlands \\ e-mail: aguilber@rssd.esa.int \\ 2 Centre for Astrophysics and Planetary Science, School of Physical Sciences, University of Kent, Canterbury CT2 7NH, UK \\ 3 Osservatorio Astrofisico di Arcetri, Largo Enrico Fermi 5, 50125 Firenze, Italy \\ ${ }^{4}$ Sterrewacht Leiden, PO Box 9513, 2300 RA Leiden, The Netherlands \\ 5 Wincor Nixdorf Portavis GmbH, Wendenstrasse 21, 20097 Hamburg, Germany
}

Received 12 May 2014 / Accepted 6 June 2014

\begin{abstract}
Context. Comets are believed to hold a relatively pristine record of the physical and chemical processes that occurred during the formation and evolution of the solar system. Thorough investigations of these small bodies, such as the one that will be performed by the ESA/Rosetta cornerstone mission, are thus supposed to bring strong and unique constraints on the origins of the solar system. Aims. Because comet 67P/Churyumov-Gerasimenko was only recently selected as the target for the ESA/Rosetta mission, there has been little opportunity to study its pre-perihelion activity. This phase is, however, very important for the mission, since the global mapping of the nucleus and the choice of landing site for Philae will be performed during this pre-perihelion phase. Here, we report previously unpublished data of the last pre-perihelion passage of this comet, observed between May and September 2008.

Methods. The gas and dust activity of comet $67 \mathrm{P} / \mathrm{Churyumov-Gerasimenko} \mathrm{are} \mathrm{studied} \mathrm{through} \mathrm{visible} \mathrm{spectroscopy} \mathrm{and} \mathrm{broadband}$ imaging, respectively, covering a range of pre-perihelion heliocentric distances between 2.99 and 2.22 AU.

Results. The data we have gathered on the dust activity are consistent with trends observed by other authors and show a strong asymmetry between the pre- and post-perihelion phases of the orbit. The spectra do not show any lines due to the emission of volatiles, and upper limits on their production rates are typically one order of magnitude lower than at the equivalent post-perihelion heliocentric distances. The asymmetry in the pre- and post-perihelion phases of the activity may be due to a dusty crust quenching the activity at the surface of 67P. We estimate that this crust could be about $12 \mathrm{~cm}$ thick, although not uniform across the surface. Even if no gas is individually detected, the coma surface brightness profiles might indicate a possible contamination from gaseous species emitted before the comet actually reaches perihelion.
\end{abstract}

Key words. comets: individual: 67P/Churyumov-Gerasimenko - methods: observational

\section{Introduction}

Comets are believed to be some of the most primitive objects in the solar system, holding crucial clues to the formation of our planetary system. By studying comets, we can access a record of the physical and chemical processes that occurred in the early phase of the solar system's evolution, thus putting constraints on its history. In this context, the ESA/Rosetta cornerstone mission is designed to perform the most thorough investigation of a comet ever. The comet selected for the 2014 is the Jupiter family comet 67P/Churyumov-Gerasimenko (hereafter 67P), which has therefore been closely studied since 2003 .

Dedicated observations started with a post-perihelion monitoring of 67P, performed after its 2002 perihelion passage (Schulz et al. 2004; Lamy et al. 2006; Lara et al. 2005). Since then, a number of studies have been performed. The inactive nucleus was studied by Tubiana et al. $(2008,2011)$ and Lowry et al. (2012), and its thermal properties were studied by Kelley et al. (2006, 2009) and Lamy et al. (2008). When active, the morphology and composition of the coma was constrained by Vincent et al. (2013) and Schleicher (2006), respectively; the dust trail was studied by Ishiguro (2008), Kelley et al. (2008),

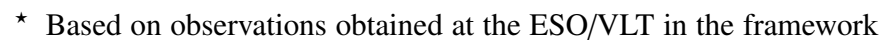
of programme 281.C-5004. and Agarwal et al. (2010); and the polarisation properties of the comet dust were studied by Hadamcik et al. (2010). The overall dust environment of the comet coma and tail was studied by Fulle et al. (2004), Moreno et al. (2004), and Fulle et al. (2010).

Because 67P was only lately selected as the ESA/Rosetta target, there was only one opportunity to study its pre-perihelion activity as it returned to perihelion in 2008 (Tozzi et al. 2011; Lara et al. 2011; Snodgrass et al. 2013). This period is, however, very important for the mission since the Global-Mapping phase and choice of a landing site for Philae will be performed at that time. In this Letter, we report previously unpublished data of this pre-perihelion passage (gas and dust activity determined from spectroscopy and broadband imaging), which correspond to exactly the phase where the ESA/Rosetta spacecraft reaches the comet so as to study the onset of its activity.

\section{Observations and data reduction}

Comet 67P was monitored from the ESO/VLT between May 29, 2008 and September 16, 2008, covering a range of pre-perihelion heliocentric distances between 2.99 and 2.22 AU. The observation of visible spectra and broadband images were spread over four runs with FORS, corresponding to $r=2.99,2.75,2.48$, and 2.22 AU. Standard calibration were provided by the ESO 
calibration plan. The basic data reduction steps were performed with the FORS pipeline procedures, applied to all images and spectra. The broadband images obtained with $B, V, R$, and $I$ filters were used to derive dust productions, given in terms of $\operatorname{Af} \rho$ (A'Hearn et al. 1989), and assuming values for the solar flux as given by Colina et al. (1996).

In addition to standard calibrations, the spectrum of the solar analogue HD 208847 was observed with the same instrumental settings as need to calibrate the comet spectra. The sky subtraction was done by scaling and taking the median of all frames. The spectra were then analysed following the procedure detailed in Schulz et al. (2004). One-dimensional spectra with a signal-to-noise ratio $S / N>100$ were extracted, and fitted with a "reddened" spectrum of the solar analogue star, excluding typical regions of cometary emission, so as to approximate the cometary continuum flux. This fit was then subtracted in order to yield the flux of the cometary emissions, which were then used to compute gas production rates, determined with the vectorial model using procedures and parameters described in Schulz et al. (1998). Some Af $\rho$ measurements were also derived from a region of the spectra $(520-525 \mathrm{~nm})$ that is known to represent a clean dust continuum.

\section{Gas activity}

Constraints on the gas activity of 67P in the pre-perihelion phase are particularly important for the ESA/Rosetta mission, since the effects of gas drag could potentially be problematic for the spacecraft large solar panels. With spectra taken at four different moments on the inbound part of the orbit, we aim at determining the moment at which gas can be detected statistically. The most abundant volatile in comets is water, which is spectroscopically inactive at any ground-accessible wavelength. The dissociation product $\mathrm{OH}$ has a line near $3080 \AA$, near the ozone atmospheric absorption edge, which also makes it difficult to observe from the ground. Therefore, we targeted one of the most sensitive limits to cometary outgassing from the ground, determined from a $\mathrm{CN}$ emission line at $3889 \AA$. Because $\mathrm{CN}$ parent molecules are more volatile than water, this additionally allows detecting earlier gas activity, triggered at a lower temperature.

The spectra obtained in 2008 are shown in Fig. 1. The typical CN emission line is absent from all spectra, even at 2.2 AU. No other gas species can be detected. Table 1 presents $3 \sigma$ upper limits to the $\mathrm{CN}$ productions rates, compared to those obtained in 2003 on the outbound part of the orbit at comparable heliocentric distances (Schulz et al. 2004). Production rates are typically one order of magnitude lower than for the same postperihelion distances. This strong asymmetry between the preand post-perihelion phases of the orbit agrees with past results (see Snodgrass et al. 2013, for a review). The lack of volatile detection may be interpreted as due to the delay in the thermal wave reaching the buried sublimation fronts, reducing the expected overall activity on the inbound part of the orbit, where the nucleus is being heated up.

\section{Dusty crust at the surface of 67P}

Although de Sanctis et al. (2005) do not foresee the formation of any permanent stable dusty crust at the surface of 67P, the asymmetry suggested by the pre- and post-perihelion activity seems a strong indication that such a crust is indeed present on at least part of the surface to quench the pre-perihelion activity. Rosenberg \& Prialnik (2009) thoroughly studied the formation

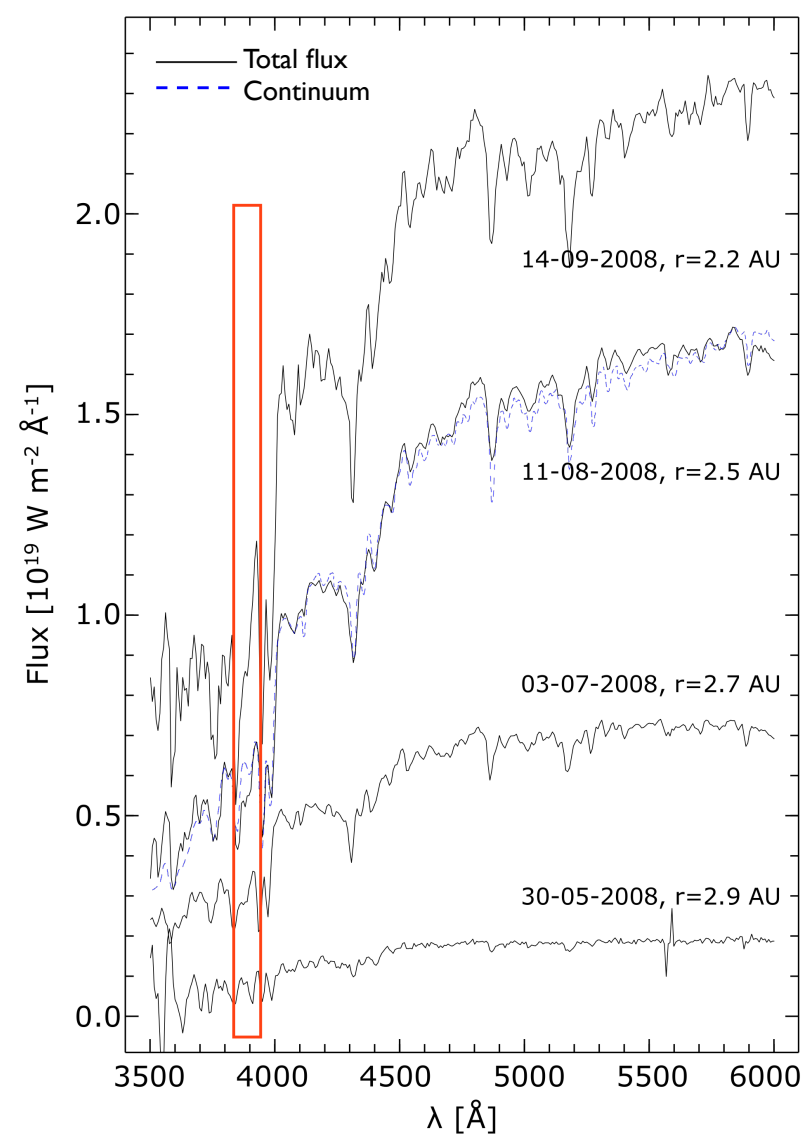

Fig. 1. Pre-perihelion co-added spectra of comet 67P, presented as flux vs. wavelength. The total flux increases from May 2008 (bottom) to September 2008 (top). The continuum is shown as a dotted line for only one spectrum. The superimposed rectangle highlights the wavelength range where we would expect to detect a $\mathrm{CN}$ emission feature: none is detected here.

Table 1. Pre- and post-perihelion CN production rates of comet 67P.

\begin{tabular}{lccccc}
\hline \hline \multicolumn{3}{c}{ Pre-perihelion } & \multicolumn{3}{c}{ Post-perihelion $^{a}$} \\
Date & $r$ & $Q(\mathrm{CN})$ & Date & $r$ & $Q(\mathrm{CN})$ \\
& $\mathrm{AU}$ & $10^{23} \mathrm{~s}^{-1}$ & & $\mathrm{AU}$ & $10^{23} \mathrm{~s}^{-1}$ \\
\hline $30-05-08$ & 2.9 & $<0.13$ & $30-04-03$ & 2.9 & $5.0 \pm 0.3$ \\
$03-07-08$ & 2.7 & $<1.19$ & & & \\
$11-08-08$ & 2.5 & $<2.95$ & $09-03-03$ & 2.5 & $24.7 \pm 5.5$ \\
$14-09-08$ & 2.2 & $<0.99$ & $11-02-03$ & 2.3 & $24.2 \pm 3.4$ \\
\hline
\end{tabular}

Notes. ${ }^{(a)}$ Values from Schulz et al. (2004).

and destruction of a potential dusty crust at the surface of 67P, which we do not intend to reproduce here. However, based on the gas production rates determined in the previous section, we aim at estimating the thickness of dust that would be required at the surface to reproduce the thermal lag suggested in the preperihelion data.

Any ice buried under a dusty crust does not sublimate freely, but follows a diffusion regime where gas escapes through the crust. The mass loss rate from such a buried layer can be written as (assuming an ideal gas law, (Fanale \& Salvail 1984; Schorghofer 2008; Gundlach et al. 2011; Guilbert-Lepoutre 2014):

$J \sim \frac{\varphi}{\Delta r} \sqrt{\frac{2 m}{\pi k_{\mathrm{B}} T}} P_{\mathrm{S}}(T)$, 
A. Guilbert-Lepoutre et al.: Pre-perihelion activity of comet 67P/Churyumov-Gerasimenko

Table 2. Maximum crust thickness encountered at the surface of 67P.

\begin{tabular}{ccr}
\hline \hline Date & $r[\mathrm{AU}]$ & $\Delta r[\mathrm{~cm}]$ \\
\hline $30-05-08$ & 2.99 & 12.0 \\
$03-07-08$ & 2.75 & 10.5 \\
$11-08-08$ & 2.48 & 9.1 \\
$14-09-08$ & 2.22 & 12.0 \\
\hline
\end{tabular}

with $\varphi$ the permeability of the crust, $\Delta r$ [m] its thickness, $m$ $[\mathrm{kg}]$ the molecular weight, $k_{\mathrm{B}}\left[\mathrm{JK}^{-1}\right]$ the Boltzmann constant, $T[\mathrm{~K}]$ the temperature, and $P_{\mathrm{S}}(T)[\mathrm{Pa}]$ the saturation vapour pressure, which is given by the Clausius-Clapeyron equation $P_{\mathrm{S}}(T)=\alpha \mathrm{e}^{-E_{\mathrm{a}} / k_{\mathrm{B}} T}$, with $\alpha=3.86 \times 10^{10} \mathrm{~Pa}$ and $-E_{\mathrm{a}} / k_{\mathrm{B}}=$ 4023.66 K for HCN, the parent molecule of $\mathrm{CN}$. The temperature is determined using a numerical model of three-dimensional heat transport (Guilbert-Lepoutre et al. 2011). The model solves the heat equation taking into account conduction via contacts between grains, radiation within pores, insolation, and thermal emission at the surface. The temperature distribution is computed as a function of time and orbital position of the comet, both at the surface and inside the nucleus. We assume that the upper layer is made of porous dust, with $85 \%$ porosity and thermal inertia of $15 \mathrm{Jm}^{-2} \mathrm{~K}^{-1} \mathrm{~s}^{-1 / 2}$ (the upper limit determined by Lowry et al. (2012), and intend to estimate its thickness $\Delta r$ by inverting Eq. (1).

We must keep in mind that $67 \mathrm{P}$ is known to present jets, so it is unlikely that the entire surface is active: we expect that this would lead to local variations in the crust thickness. However, the spectra were observed over a fraction of the comet rotation phase, sometimes quite long, which makes it almost impossible to identify a specific active area. We have therefore considered several cases to estimate the area of active surface. First, we assumed that the nucleus is a sphere, with the overall surface being active. Second, we used the total nucleus area from the shape model provided by Lowry et al. (2012). Then, we considered the total area illuminated during the spectroscopic observations. We find that the active area has a limited effect on the crust thickness compared to other parameters, such as the thermal conductivity or the orientation of the spin axis. For instance, we find that $\Delta r$ varies strongly with the latitude at the surface of 67P, and thus also with the orientation of the spin axis. For example, if we assume the axis is perpendicular to the orbital plane, $\Delta r$ can vary from $1 \mathrm{~mm}$ at the poles to a dozen centimetres at the equator. For reference, we present in Table 2 the maximum crust thickness encountered on the surface of $67 \mathrm{P}$ in our simulations.

We stress again that the overall surface is not necessarily covered by the same amount of dust. Rosenberg \& Prialnik (2009) even suggest that 67P surface is not entirely covered by dust at all, based on comparing of post-perihelion water production rates with those computed by their model with and without dusty crust. We estimate that the maximum crust thickness is $\sim 12 \mathrm{~cm}$, which is consistent with the crusts of several centimetres found by de Sanctis et al. (2005), Kossacki \& Szutowicz (2006), and Rosenberg \& Prialnik (2009) based on water production rates.

\section{Dust activity}

The dust production of 67P is approximated in terms of $\operatorname{Af} \rho$, which provides useful constraints to compare the comet to others and to monitor its activity along its orbit (see Table 3). However, these measurements must be treated with caution, since the use of $\operatorname{Af} \rho$ assumes a steady state coma (i.e. a symmetric coma
Table 3. Pre-perihelion dust production of 67P in terms of Af $\rho$ values.

\begin{tabular}{lcccccc}
\hline \hline \multirow{2}{*}{ Date } & $r$ & \multicolumn{4}{c}{$\operatorname{Af} \rho^{a}[\mathrm{~cm}]$} \\
& {$[\mathrm{AU}]$} & $B$ & $V$ & $R$ & $I$ & Spec. $^{b}$ \\
\hline $30-05-08$ & 2.9 & $11 \pm 2$ & $19 \pm 1$ & $18 \pm 2$ & & $4 \pm 1$ \\
$03-07-08$ & 2.7 & $23 \pm 2$ & $33 \pm 1$ & $37 \pm 1$ & $31 \pm 3$ & $11 \pm 1$ \\
$11-08-08$ & 2.5 & $49 \pm 2$ & $63 \pm 2$ & $73 \pm 2$ & $68 \pm 3$ & $23 \pm 1$ \\
$14-09-08$ & 2.2 & $45 \pm 1$ & $62 \pm 2$ & $73 \pm 1$ & $65 \pm 1$ & $31 \pm 1$ \\
\hline
\end{tabular}

Notes. ${ }^{(a)}$ At $10^{4} \mathrm{~km}$ from the comet and corrected for zero phase angle.

(b) Derived from the $520-525 \mathrm{~nm}$ region in spectra.

produced by a constant source, emitting grains moving with a constant velocity away from the nucleus, and with constant scattering properties), an assumption that we know not to apply to 67P (Tozzi et al. 2011; Snodgrass et al. 2013). These measurements are nonetheless useful for gaining direct access to the dust column density along the line of sight and to the processes dominating the evolution of the dusty coma once the grains are emitted. A steady-state coma is expected to have a linear surface brightness profile, with a slope of -1 on a logarithmic scale. If effects of the solar radiation pressure are accounted for, this slope should be -1.5 (Jewitt \& Meech 1987). Several other processes can affect the surface brightness profiles and produce slopes that are different from these values, such as the sublimation of icy grains, the contamination from gases, or a variable mass loss from the nucleus. The surface brightness profiles for $67 \mathrm{P}$ are plotted in Fig. 2. They have been fitted on their linear part, with the innermost pixels left out: the corresponding slopes are presented in Table 4. Consistent values were published by Snodgrass et al. (2013), based on the same $R$-band observations but with different data reduction and calibration. Our Af $\rho$ values are also consistent with those published by Fulle et al. (2010).

Observations performed in the $R$-band are sensitive to the reflected sunlight from the dust and are not significantly affected by emission lines from any gaseous species in the coma. They therefore provide very good access to dust production. In the $R$ band, the slope of the surface brightness profile decreases with the heliocentric distance. Slopes computed for $V$-band observations show similar behaviour. These observations could be affected by $\mathrm{C}_{2}$ emissions, but because: (i) the trend is very similar to the one in the $R$ band; (ii) no $\mathrm{C}_{2}$ line can be detected in the spectra; and (iii) 67P is depleted in $C_{2}$ (Schulz et al. 2004) according to the classification scheme introduced by A'Hearn et al. (1995), we can assume that there should only be very limited contamination from this volatile. The trend is not shared by the $B$-band observations, either because of a lower signalto-noise ratio in this filter or because $B$-band observations can potentially be affected by emission lines of $\mathrm{CN}, \mathrm{C}_{3}$, and $\mathrm{C}_{2}$. We should also mention that the $\operatorname{Af} \rho$ parameter and the dust mass loss rate would follow the same time dependence only in the unlikely event that both the dust size distribution and grain ejection velocities do not depend on time. Given the curvature of the brightness profiles, particularly in the $B$ band, we suspect that our results could indicate gas contamination and could thus be consistent with gas being emitted already at large heliocentric distances, although at such low levels that no gaseous species can be detected individually in the corresponding spectra.

\section{Summary}

We present previously unpublished data on the pre-perihelion dust and gas activity of comet $67 \mathrm{P} /$ Churyumov-Gerasimenko, the target of the ESA/Rosetta cornerstone mission. Our results 


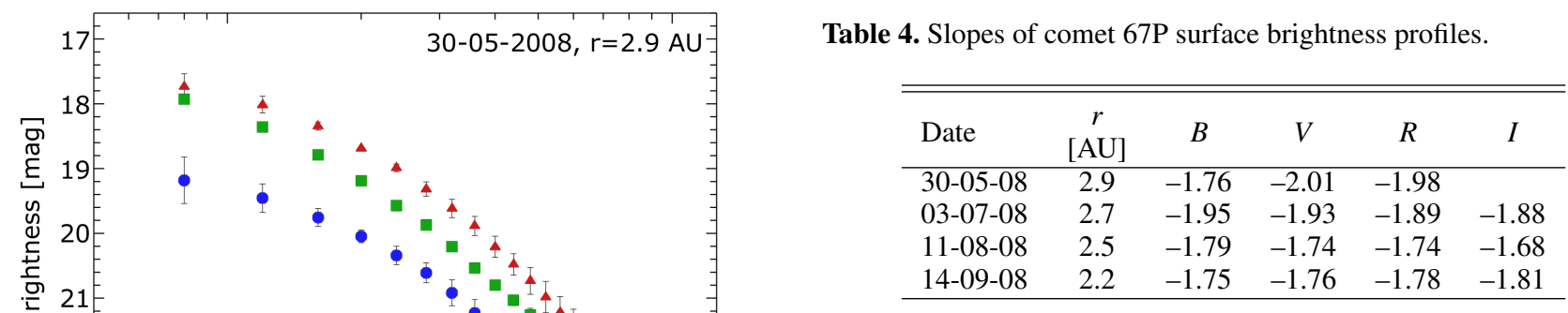

can be summarised in three main points. The spectra lack emission lines caused by the sublimation of ices, even at $2.2 \mathrm{AU}$. The associated $\mathrm{CN}$ production rates are typically one order of magnitude lower than at comparable post-perihelion distances. The asymmetry suggested by this data is consistent with previous studies and may be interpreted as due to the presence of a dusty porous crust quenching the surface activity. Based on thermal considerations, we estimate that this crust could be as thick as $12 \mathrm{~cm}$, so to reproduce the $\mathrm{CN}$ production rates, although it might not be uniform across the surface. From the surface brightness profiles obtained in different filters, we suspect that our data are consistent with gas being emitted before perihelion, although at such low levels that no gaseous species can be detected through individual emission line in the corresponding spectra.

Acknowledgements. A.G.L. acknowledges support from the ESA Fellowship programme.

\section{References}

A'Hearn, M. F., Campins, H., Schleicher, D. G., \& Millis, R. L. 1989, ApJ, 347, 1155

A'Hearn, M. F., Millis, R. L., Schleicher, D. G., Osip, D. J., \& Birch, P. V. 1995, Icarus, 118, 223

Agarwal, J., Müller, M., Reach, W. T., et al. 2010, Icarus, 207, 992

Colina, L., Bohlin, R. C., \& Castelli, F. 1996, AJ, 112, 307

de Sanctis, M. C., Capria, M. T., \& Coradini, A. 2005, A\&A, 444, 605

Fanale, F. P., \& Salvail, J. R. 1984, Icarus, 60, 476

Fulle, M., Barbieri, C., Cremonese, G., et al. 2004, A\&A, 442, 357

Fulle, M., Colangeli, L., Agarwal, J., et al. 2010, A\&A, 522, A63

Gundlach, B., Skorov, Y. V., \& Blum, J. 2011, Icarus, 213, 710

Guilbert-Lepoutre, A. 2014, Icarus, 231, 232

Guilbert-Lepoutre, A., Lasue, J., Federico, C., et al. 2011, A\&A, 529, A71

Hadamcik, E., Sen, A. K., Levasseur-Regourd, A. C., Gupta, R., \& Lasue, J. 2010, A\&A, 517, A86

Ishiguro, M. 2008, Icarus, 193, 96

Jewitt, D., \& Meech, K. J. 1987, ApJ, 317, 992

Kelley, M. S., Woodward, C. E., Harker, D. E., et al. 2006, ApJ, 651, 1256

Kelley, M. S., Reach, W. T., \& Lien, D. J. 2008, Icarus, 193, 572

Kelley, M. S., Wooden, D. H., Tubiana, C., et al. 2009, AJ, 137, 4633

Kossacki, K. J., \& Szutowicz, S. 2006, Planet. Space Sci., 54, 15

Lamy, P. L., Toth, I., Weaver, H. A., et al. 2006, A\&A, 458, 669

Lamy, P. L., Toth, I., Groussin, O., et al. 2008, A\&A, 489, 777

Lara, L. M., de León, J., Licandro, J., \& Gutiérrez, P. J. 2005, Earth Moon Planets, 97, 165

Lara, L. M., Lin, Z.-Y., Rodrigo, R., \& Ip, W.-H. 2011, A\&A, 525, A36

Lowry, S., Duddy, S. R., Rozitis, B., et al. 2012, A\&A, 548, A12

Moreno, F., Lara, L. M., Munoz, O., Lopez-Moreno, J. J., \& Molina, A. 2004, ApJ, 613, 1263

Rosenberg, E. D., \& Prialnik, D. 2009, Icarus, 201, 740

Schleicher, D. G. 2006, Icarus, 181, 442

Schorghofer, N. 2008, ApJ, 682, 697

Schulz, R., Arpigny, C., Manfroid, J., et al. 1998, A\&A, 335, L46

Schulz, R., Stüwe, J. A., \& Boehnhardt, H. 2004, A\&A, 422, L19

Snodgrass, C., Tubiana, C., Bramich, D. M., et al. 2013, A\&A, 557, A33

Tozzi, G. P., Patriarchi, P., Boehnhardt, H., et al. 2011, A\&A, 531, A54

Tubiana, C., Barrera, L., Drahus, M., \& Boehnhardt, H. 2008, A\&A, 490, 377

Tubiana, C., Boehnhardt, H., Agarwal, J., et al. 2011, A\&A, 527, A113

Vincent, J.-B., Lara, L. M., Tozzi, G. P., Lin, Z.-Y., \& Sierks, H. 2013, A\&A, 549, A121 